\title{
UN PROGRAMA QUE "NO PUEDE CONFORMAR A TODOS”: ECONOMÍA Y BUROCRACIA EN LOS AÑOS FINALES DEL PRIMER PERONISMO
}

\section{A PROGRAM THAT "CANNOT CONFORM TO ALL": ECONOMICS AND BUREAUCRACY IN THE FINAL YEARS OF THE FIRST PERONIST}

\author{
Marcelo Rougier \\ Universidad de Buenos Aires, Buenos Aires, Argentina, <marcelorougier@yahoo.com.ar>
}

\author{
Martín Stawski \\ Universidad Nacional de Tres de Febrero, Buenos Aires, Argentina, \\ $<$ martinstawski@hotmail.com>
}

Resumen. El presente artículo indaga en algunos aspectos de la política económica del gobierno peronista en el periodo que se abre a partir de 1953-1954, al momento de consolidación de los cambios introducidos a las políticas económicas iniciales luego de la crisis de 1949-1952. Específicamente se estudia un programa poco considerado por la historiografía, el Plan de Acción de Equilibrio para la Economía Nacional anunciado en octubre de 1954 y las transformaciones burocráticas que lo acompañaron. De esta forma, nuestro objetivo es anudar el análisis de las modificaciones operadas en los ámbitos de gestión económica con las medidas tomadas en materia de política económica e identificar las capacidades estatales e instrumentos diseñados por el peronismo.

Palabras clave: peronismo; planificación; burocracia; política económica; elites estatales.

\begin{abstract}
This article explores some aspects of the Peronist government's economic policy in the period from 1953 to 1954 at the time of consolidation of the changes made to the initial economic policy after the crisis of 1949-1952. Specifically we study a program scarcely considered by the historians, the Action Plan for National Economic Balance of October 1954, and bureaucratic changes that accompanied it. Thus, our goal is to tie the analysis of the amendments made in the areas of economic management with the measures taken in regard to economic policy, with the specific purpose of identifying the links between the two areas and arrive at a more complete understanding of actions and determinants of these changes.
\end{abstract}

Key words: peronism; planning; bureaucracy; economic policy; state elites.

Fecha de recepción: marzo de 2013. Fecha de aceptación: julio de 2013.

Am. Lat. Hist. Econ., año 21, núm. 1, enero-abril, 2014, pp. 174-199 


\section{INTRODUCCIÓN}

$\mathrm{L}$

os gobiernos de Juan Perón de 1946 a 1955 no pueden ser vistos ya de manera monolítica. En las últimas décadas numerosas investigaciones han dado cuenta de modificaciones importantes, al menos claramente en la dinámica política, social y económica, durante el transcurso del "decenio" peronista que alientan a identificar etapas o periodos con características específicas. ${ }^{1}$ En este sentido es posible discernir cambios notables a partir de 1949-1952, tanto en las políticas económicas como en la estructura burocrática respecto a los primeros años de gestión.

Ya desde el inicio de la nueva gestión el presidente, los ministros y los secretarios procuraron dar entidad legal a las transformaciones que se habían desarrollado en los años previos al surgimiento del peronismo, y continuaron con la creación de nuevas agencias estatales, principalmente de gestión económica. ${ }^{2}$ Este trayecto tuvo su génesis con la reforma financiera de 1946 encabezada por el multifacético empresario Miguel Miranda. ${ }^{3}$

Prontamente, el llamado "mago de las finanzas" se transformó en el principal hacedor de la política económica, ya que no sólo disponía de un poder superlativo en relación con la política interna -con el control del Banco Central y del Consejo Económico Nacional-, sino que también manejaba el comercio exterior a través del Instituto Argentino para la Promoción del Intercambio (en adelante IAPI). ${ }^{4} \mathrm{El}$ mando de estas instituciones le permitió a Miranda un margen de maniobra inédito hasta ese momento de la política económica argentina.

Sin embargo, algunas disposiciones de gobierno poco claras que vinculaban diversos emprendimientos empresariales con el propio Miranda y variadas denuncias de corrupción sobre su desempeño como funcionario público provocaron su caída a inicios de enero de 1949; por miedo a un atentado Miranda se exilió en Montevideo, donde falleció en medio de un profundo ostracismo pocos años después. ${ }^{5}$ Sin embargo, más allá de esos avatares que lo vinculaban personalmente, la partida del ex hombre

${ }^{1}$ Un trabajo pionero en ese sentido es el de Ferrer, Crisis, 1977. También Gerchunoff y Llach, Ciclo, 1998; Rapoport, Historia, 2000; Brennan y Rougier, Politics, 2009, y Rougier, Mercado, 2011, y Economía, 2012, han identificado, si bien con diferencias, distintas etapas en la evolución macroeconómica y en la política económica. En el plano político véanse Berrotarán, Plan, 2003, y "Estado", 2008; Prol, "Peronismo", 2003; Jáuregui, "Prometeo", 2004; Belini, Industria, 2009, y Stawski, “Consejos", 2012, y "Equipo”, 2012.

${ }^{2}$ Campione, Prolegómenos, 2003, y Berrotarán, Plan, 2003. jos", 2012.

${ }^{3}$ Sobre la trayectoria de Miranda véanse, entre otros, Rein, Juan, 2006, y Stawski, "Conse-

${ }^{4}$ El IAPI fue una institución clave dentro de la política económica del peronismo con diferentes atribuciones durante toda la etapa, aunque dedicada principalmente al monopolio del comercio exterior. Al respecto véase, entre otros, Novick, IAPI, 1986.

${ }^{5}$ Rein, Juan, 2006. 
fuerte de la economía coincidió con una doble circunstancia: la crisis económica de 1949 y la reforma constitucional, situación que alentó la transformación de la estructura burocrática tendente a mejorar los mecanismos y la calidad de la intervención pública.

La sanción de una nueva constitución -sostenida en el derecho constitucional liberal y sin alterar de raíz el sistema político- modificó la composición de las carteras nacionales que pasaron de ocho a 19. En materia de competencia económica se crearon tres nuevos ministerios que se sumaron al longevo Hacienda: Economía, Industria y Comercio, y Finanzas. Y sería desde este último que surgiría la figura excluyente de decisión económica del peronismo hasta su derrocamiento: Alfredo Gómez Morales.

Esas transformaciones fueron acompañadas, una vez que se presentó la crisis, por una redefinición de las políticas económicas encaradas hasta ese entonces y de las formas de organización de la coordinación y conducción de esas políticas. En efecto, luego de una fuerte intervención y de aliento a la industria y el consumo, la política económica debió virar a resolver los problemas de escasez de divisas y de deterioro de la situación fiscal apelando a diversas estrategias que en términos generales supusieron estímulos a las actividades agropecuarias y al ingreso del capital extranjero, un freno al consumo y mayor austeridad en el gasto público. Muestra cabal de este novedoso escenario fue la sanción del primer programa de estabilización ortodoxo dentro de la dinámica de la industrialización por sustitución de importaciones en Argentina: el Plan de Acción en Materia Económica de 1949, que revocaba en cierta medida los objetivos iniciales del gobierno peronista. ${ }^{6}$ Más tarde se sumó un nuevo y más radical programa de ajuste, a comienzos de 1952, cuando las condiciones económicas, principalmente en el sector externo, se agravaron.

Una vez encaradas estas políticas se continuó con la reforma estatal y paralelamente al anuncio ante la prensa del segundo plan quinquenal en el mes de junio de 1952 se produjo la segunda reforma ministerial en el peronismo; y, dentro de ella, los organismos económicos iban a sufrir nuevamente modificaciones sustanciales; se creó la Secretaría de Estado Ministerio de Asuntos Económicos y se sustituyó el Ministerio de Economía por el de Comercio Exterior. La restructuración profundizaba la necesidad del poder ejecutivo de centralizar en pocos organismos y personas las decisiones de índole económica y financiera con el fin de tener un control más estricto sobre la política económica. En otras palabras, el cambio de estrategia económica suponía necesariamente una adecuación de la estructura burocrática que garantizara su éxito.

\footnotetext{
${ }^{6}$ Véanse Rougier, Economía, 2012, y Stawski, “Consejos”, 2012.
} 
Significativamente, la literatura heredada no ha abordado de manera detallada los últimos años de la economía peronista y menos examinado las alteraciones producidas a escala económica y en los organismos de gestión de manera conjunta. Este artículo indaga precisamente en el periodo que se abre a partir de 1953-1954, cuando los efectos más duros de la crisis habían pasado y era necesario redefinir la estrategia económica inicial y tornarla sustentable en el más largo plazo.

Nuestro objetivo es anudar en el análisis las modificaciones operadas en los ámbitos de gestión económica con las medidas tomadas en materia de política económica, con el propósito específico de identificar las articulaciones entre las dos esferas y arribar a una comprensión más acabada de las acciones y determinantes de esas transformaciones. Adicionalmente, el trabajo aporta a identificar la generación de capacidades estatales e instrumentos para la estructuración de determinadas estrategias económicosociales y su consecución.

Sobre la base de fuentes primarias (documentos oficiales y publicaciones periódicas principalmente), en la primera parte abordamos el análisis del Plan de Acción de Equilibrio para la Economía Nacional ${ }^{7}$ anunciado en octubre de 1954 y sus implicancias para el nuevo contexto económico abierto con la recuperación económica luego de los duros años de crisis entre 1949 y 1952. En un segundo momento analizamos los cambios ocurridos con la reforma ministerial de junio de 1954 en las distintas dependencias relacionadas con la coordinación económica. ${ }^{8}$ Finalmente, las últimas reflexiones están destinadas a identificar los vínculos entre ambos procesos, considerados parte sustancial de la consolidación de la estrategia política y económica del peronismo en sus últimos años de gobierno.

\section{El Plan de Acción de Equilibrio para la Economía Nacional DE 1954}

Entre 1946 y 1948 la economía argentina tuvo un muy buen desempeño, especialmente del sector industrial, mientras los salarios de los trabajadores se incrementaban de manera notable. Ello fue consecuencia de la recuperación del comercio internacional a la salida de la guerra y del conjunto

\footnotetext{
${ }^{7}$ A este conjunto de medidas también se lo conoció oficialmente como Plan de Acción para el Equilibrio de la Economía Nacional, pero en este caso decidimos utilizar el nombre que mencionamos más arriba.

${ }^{8}$ Este análisis que indaga en la noción de calidad estatal se apoyará en términos teóricos en Oszlak, Politicas, 1980, y Teorías, 1984. También se pueden consultar, entre otros, Schneider, "Relaciones”, 1999 y, especialmente, Castellani y Llanpart, "Debates”, 2012.
} 
de instrumentos diseñados por el gobierno peronista en sus años iniciales. ${ }^{9}$ No obstante, el panorama del comercio exterior era muy complicado a la salida de la segunda guerra mundial por la falta de divisas convertibles de los países compradores de los productos argentinos. La ayuda económica y financiera que Estados Unidos promovió a través del plan Marshall alentó las colocaciones desde ese país y desplazó a Argentina de sus mercados internacionales. Este hecho sumado al incremento de las importaciones que demandaba una industria en fuerte crecimiento se tradujo en la aparición de saldos negativos en la balanza comercial y el agotamiento de las reservas.

El gobierno respondió con el desplazamiento de Miguel Miranda por Gómez Morales y una serie de políticas de ajuste que pretendieron mejorar las condiciones en las que se desempeñaban las actividades agropecuarias (mejora de los precios de sus productos) y de políticas restrictivas tendentes a contener el incremento de los precios (que había comenzado a preocupar a las autoridades desde fines de 1948).

Estas medidas quedaron enmarcadas en un denominado plan de acción en materia económica, lanzado en julio de 1949, cuyo principal objetivo era mantener la estabilidad de precios y "racionalizar" la estructura productiva; esto es, tratar de generar una mayor armonía entre las actividades agropecuarias (hasta entonces discriminadas) y las industriales (hasta entonces promovidas fuertemente a través de la política salarial y crediticia). ${ }^{10}$

Pero las circunstancias negativas se mantuvieron en los años siguientes, más allá de alguna pequeña mejora, determinadas principalmente por la evolución del comercio exterior y los precios internacionales. Una pésima cosecha a fines de 1951, además, complicó el panorama del sector externo. Por otro lado la caída del salario real desde 1949 era también un problema a resolver, más cuando la inflación anual superaba holgadamente el 30\%. Para restablecer el equilibrio de precios y salarios perdido, el gobierno convocó a los representantes de los trabajadores y de los empresarios. Mientras los delegados obreros reclamaban una recomposición del salario, los industriales sostenían la necesidad de bajar los costos de producción aumentando la productividad obrera.

En parte, como resultado de esa compulsa, el gobierno lanzó un plan de estabilización en febrero de 1952 que aumentaba y congelaba los salarios por dos años; también se profundizaron las políticas fiscales y monetarias restrictivas tendentes a contener el proceso inflacionario. En rigor, el

\footnotetext{
${ }^{9}$ Rougier, Economía, 2012, pp. 100-107.

${ }^{10}$ A pesar de que en la faz discursiva el peronismo intentó mostrarse disruptivo con las políticas económicas de la década de 1930, es interesante destacar que el gobierno de Juan Perón, al momento de crear planes económicos de corto plazo, utilizó la misma denominación que el Plan de Acción Económica Nacional de 1933. Así sucedió con los llamados Plan de Acción en Materia Económica de 1949 y el Plan de Acción de Equilibrio para la Economía Nacional de 1954.
} 
nuevo ordenamiento no representaba una innovación en materia de política económica, sino una sistematización y extensión de las medidas que se venían aplicando desde fines de 1948 y constituiría un primer paso hacia la aplicación de otras de carácter más permanente que sólo podrían implantarse una vez doblegados los problemas de la crítica coyuntura de 1952.

Hacia 1953 quedaba claro que el plan de ajuste había sido exitoso, la inflación había caído considerablemente y la recuperación de la economía estaba en marcha. Con la aplicación del segundo plan quinquenal y de la Ley de Inversiones Extranjeras en ese mismo año, el gobierno pretendía impulsar la producción agropecuaria y avanzar en la integración de las actividades industriales, especialmente en la producción de insumos y equipos cuya importación pesaba gravemente sobre las cuentas externas. En este sentido se trataba de una apuesta a la inversión (interna y externa) de modo que pudiera profundizarse la sustitución de importaciones (ahora de bienes más complejos) y con ello garantizar las condiciones para sostener el crecimiento económico.

No obstante, uno de los problemas irresueltos estaba referido a la implementación de reformas en la organización de los procesos de trabajo tendentes a incrementar la productividad, que estaba estancada desde 1948. A comienzos de 1954 comenzaron los pedidos sindicales para incrementar los salarios y se registró una nueva oleada de protestas obreras que triplicó las huelgas registradas en el periodo 1950-1953 y que además incluyó trabajo a reglamento o huelgas de brazos caídos. ${ }^{11}$

La presión sindical para lograr una recuperación del salario real tuvo algunos resultados favorables, pero simultáneamente el empresariado aumentaba los precios debido al aumento de costos de mano de obra. En este escenario volvía a aparecer la temida espiral inflacionaria que tantos problemas había traído en los años precedentes.

Paralelamente, el gobierno convocó a los trabajadores y a los empresarios para la renegociación de los convenios colectivos fijados en el plan de 1952 y manifestó su intención de no intervenir en el establecimiento de las nuevas escalas salariales. Según el discurso oficial, los incrementos salariales sólo podían concretarse a partir de los aumentos en la productividad del trabajo; el gobierno no asistiría las demandas al movimiento obrero organizado como lo había hecho durante los años iniciales. Se trataba de un retiro del Estado en este campo que fortalecía a los empresarios, a la vez que delegaba en ellos la capacidad para contener las demandas sindicales. Esa idea entroncaba con una nueva concepción respecto a la participación del Estado manifestada por el propio Perón poco antes:

${ }^{11}$ Fernández, Huelga, 2005, y Schiavi, “Algunas”, 2011. 
Nosotros no somos ni intervencionistas ni anti intervencionistas; somos realistas. El que se dice intervencionista no sabe lo que dice; hay que ubicarse de acuerdo a las circunstancias. Las circunstancias imponen la solución. No hay sistemas, ni métodos, ni reglas de economía en los tiempos actuales. Hay soluciones concretas frente a un problema también concreto. Resuelto ese problema se va a presentar otro quizás también diametralmente opuesto al anterior. A este le daremos una solución contraria al anterior, pero no por sistema, sino por inteligente apreciación y reflexión del caso concreto. ${ }^{12}$

El nuevo problema era en este caso la renegociación de los salarios que podían salirse del rumbo previsto e impactar negativamente en los precios. El gobierno impulsó, junto a la Confederación General del Trabajo y a la Confederación General Económica, un primer congreso de organización y relaciones del trabajo, el cual se llevó a cabo entre el 23 y el 28 de agosto de 1954, con el propósito de lograr avances en la mejora de la productividad laboral.

Para los empresarios el principal objetivo consistía en reducir la excesiva influencia de los sindicatos en el lugar de trabajo y restaurar así el equilibrio de poder. En su discurso de inauguración, José Gelbard, el líder empresarial, declaró que el propósito de la conferencia era alcanzar la "racionalización humanizada" de la fábrica, necesaria para un aumento de la productividad que compensara el incremento de los salarios, el cual significaba una carga cada vez mayor para el sector empresarial. ${ }^{13}$ Pero de esas sesiones no se obtuvieron resultados concretos.

El gobierno resolvió entonces instaurar una serie de medidas a través de un denominado Plan de Acción de Equilibrio para la Economía Nacional de octubre de 1954, apoyado ahora, como veremos, en la solidez burocrática que le proporcionaría una nueva estructura ministerial. El plan no sólo suponía garantizar la estabilidad de precios, sino también un cambio importante en la participación que el Estado tenía hasta entonces en la economía y también un cambio respecto a la concepción económica que identificaba al peronismo. ${ }^{14}$ Poco antes Perón había expresado en la Primera Convención de la Confederación General Económica que

Felizmente, estamos preparando el pasaje de una organización estatal a una organización privada. El Estado argentino, dentro de nuestro concepto, se sentirá muy feliz el día que no tenga una sola empresa comercial, industrial o de la producción en su poder, porque habrá llegado el momento en que todas las empresas de la

\footnotetext{
${ }^{12}$ Gerchunoff y Llach, Ciclo, 1998, p. 171.

${ }^{13}$ Brennan y Rougier, Politics, 2009.

${ }^{14}$ Rougier, Economía, 2012, p. 185.
} 
producción, de la transformación y de la distribución que están hoy en poder del Estado, en situación floreciente, sean absorbidas por el interés privado. Esa es nuestra orientación. ${ }^{15}$

El proyecto se presentó por el propio Perón en el Salón Blanco de la Casa de Gobierno el 1 de octubre y se transmitió a todo el país por el sistema de radiotelefonía. La lógica de ejecución del plan era reforzar la política económica peronista, descrita por el elenco oficial como un importante engranaje que tenía sus antecedentes en los dos planes quinquenales y el plan de emergencia de $1952 .{ }^{16}$

El programa fue anunciado por el presidente como un programa "de corto plazo", a diferencia de los planes "de largo alcance" como los quinquenales de 1947 y 1953. Según el gobierno eran tiempos favorables para sancionar el programa, pues el pueblo estaba en condiciones de ayudar a que dicha política se materializara gracias a la experiencia adquirida en los años previos de planificación. Para Perón el objetivo del plan era consolidar el nuevo nivel de equilibrio de precios y salarios requiriendo la colaboración de todos los actores sociales.

El programa convocaba a la racionalización empresarial y a que los obreros se esforzaran por aumentar su productividad. El Estado también asumía el compromiso de estimular el mayor rendimiento de la mano de obra y dirigir la política crediticia en función de los índices de productividad que lograsen las empresas. También se llamaba a los empresarios y trabajadores a que controlasen el latente peligro inflacionario mediante el análisis de los costos de producción y los efectos que provocaban sobre ellos los convenios colectivos de salarios. ${ }^{17}$

Si bien estos objetivos eran centrales dentro de la nueva lógica de la política económica, el plan no se reducía solamente a estos aspectos. En efecto, como señalamos, lo que en realidad se estaba redefiniendo era la participación del Estado y el vínculo con los actores económicos. De hecho, un documento destinado a impulsar los contenidos del programa aporta elementos que permiten vislumbrar objetivos adicionales. ${ }^{18}$

En adelante el Estado debía ser básicamente un "orientador" de las actividades económicas, donde el éxito dependía de la "acción coordinada de las fuerzas organizadas del pueblo". La acción estatal era entendida como de "colaboración" con las actividades de los distintos sectores e incluía políticas respecto al comercio y abastecimiento externo, la racionalización de

${ }^{15}$ Perón, "Primera", 2002, p. 295.

${ }^{16}$ Perón, Plan, 1954. Para el programa económico de 1952 véanse Rapoport y Spiguel, Estados, 1994, y Rougier, Economía, 2012.

${ }^{17}$ Bitrán, Congreso, 1994.

${ }^{18}$ Perón, Plan, 1954. 
las industrias y del comercio interno. En este sentido, el documento hace referencia en primer lugar al crédito, priorizando el apoyo al sector agrario y a las cooperativas y el mejoramiento técnico de las industrias.

En segundo lugar, el texto plantea una serie de cuestiones sobre el ahorro: se reconocía que si bien el pueblo ahorraba,

aún sigue incontrolado en cuanto a gastos superfluos, que no tienen ningún valor para el vendedor ni para el consumidor, sino el de un desafío al porvenir. En consecuencia es imprescindible que todos los gobiernos (provinciales) intensifiquen la prédica del ahorro en todas sus formas para que el pueblo haga hábito de que no se trata de dar menos o más, sino de gastar con conciencia, para no sustraer algo que no se consumirá en perjuicio de lo que necesita la comunidad para el equilibrio de sus bienes. ${ }^{19}$

De esta forma la lógica mediadora e intervencionista bien característica de los primeros años de gobierno le dejaba el campo abierto a un Estado "coordinador" que propiciaba el consumo racional y el aumento de la producción. Esta idea fue materializada por el presidente de la siguiente manera: "la economía que yo puedo darles señores es una economía organizada y conducida equilibradamente. Lo demás debe hacerlo el pueblo, los empresarios, los consumidores y los obreros." ${ }^{20}$

$\mathrm{Y}$ fueron los trabajadores los que ocuparon un lugar privilegiado en el discurso de Perón, ya no para incentivarlos y defenderlos, sino para reprenderlos indirectamente por los constantes pedidos de incrementos salariales. Para el primer mandatario se habían gestado pequeños problemas que en la calle se habían "agrandado" o que de un pequeño inconveniente se trataba de realizar una gran perturbación.

Manifestaba Perón que había un aprovechamiento político de algunas situaciones, y puso como ejemplo el caso del gremio de los panaderos, quienes mediante los acuerdos laborales lograron un aumento salarial, situación que derivó en un incremento de 20 centavos en el kilogramo de pan. Sin embargo, el presidente cuestionó los convenios realizados autónomamente entre empresarios y trabajadores y criticó que estos no quisieran trabajar de noche. Así Perón dejó en falta a los trabajadores ante la sociedad y lo manifestó de la siguiente manera:

Por lo tanto, en vez de salir al amanecer, el pan sale a las nueve de la mañana. Ello ha sido motivo para que algunos se quejen y protesten. Lo cierto es que no se puede conformar a todos. Por otra parte yo no puedo obligar a la gente a trabajar de noche

${ }^{19}$ Ibid., p. 23.

${ }^{20}$ Perón, “Salón”, 2002, p. 430. 
si ellos con mucha justicia y verdad, dicen que el trabajo nocturno es insalubre [...] Otra dificultad sería que los panaderos decidieron no abrir los lunes. Ellos resolvieron cerrar un día por semana. En mi opinión personal, no está del todo bien que se cierre. Se podrían hacer turnos como en las farmacias. ${ }^{21}$

Perón observaba la misma situación en la denuncia acerca del desabastecimiento temporal de carnes. El gobernante defendía esa coyuntura y sostenía que en determinadas épocas había poca faena debido al peso de los animales, pero que en su defecto esa situación era beneficiosa, y afirmaba con su clásica retórica:

Creo que esto es muy bueno, porque así le damos descanso al hígado de los argentinos, lo que hace mucha falta. Ya que somos tan poco obedientes al médico que siempre nos dice que no comamos tanta carne, resulta muy útil que sea la naturaleza misma la que contribuya a la defensa de nuestra propia salud [...] Como ven ustedes, la gravedad del problema de la carne es terrible en la República Argentina; pero es terrible, porque comemos demasiada carne. Bien señores ¿para qué voy a seguir extendiéndome en este problema? Es suficiente andar por las calles de Buenos Aires para ver que todos son gordos. Podemos quejarnos de cualquier cosa, pero no de que no comemos. ${ }^{22}$

Previo a la exposición formal del plan, Perón explicó cómo se debía equilibrar el presupuesto nacional y declaró que se estaba en la tarea de realizar un ajuste sustancial de los gastos públicos, política indispensable para corregir los errores que se ejecutaron en el pasado en relación con las partidas presupuestarias. Como se consideraba innecesario el aumento impositivo o la emisión monetaria se resolvió achicar el gasto público, medida que, de haberse propuesto años atrás, hubiese aparejado un alto costo político. Asimismo el mandatario señaló que el programa requería de la colaboración de todos los actores económicos y delimitó cuáles deberían ser las responsabilidades de cada uno de ellos a partir de la sanción del plan.

En primer lugar, Perón señaló que en Argentina se había llegado a la máxima expresión en la justicia redistributiva, el salario real de los obreros no podía incrementarse más salvo que se produjera una mayor producción. Sobre esta base el presidente les solicitó colaboración a los empresarios, quienes debían mejorar la calidad de los productos y aumentar la producción y reducir el costo de estos. Para lograr los objetivos propuestos el presidente instó a la racionalización empresaria y a adoptar técnicas de modernización de los procesos productivos. Asimismo se les

${ }^{21}$ Ibid., las cursivas son nuestras.

${ }^{22}$ Ibid. 
encomendó la racionalización de la comercialización para disminuir los costes de distribución.

Paralelamente, Perón les solicitó que constituyesen sociedades anónimas con su correspondiente cotización bursátil para liberarse del préstamo bancario como sistema para incrementar el capital social de las empresas. ${ }^{23}$ La idea subyacente, expresada con más claridad por Miguel Revestido, ministro de Finanzas, era que el Estado no avanzase más sobre el mercado y particularmente sobre el mercado de capitales, donde la intervención se había desarrollado en la etapa inicial a través de los mecanismos creados por la reforma financiera de 1946 y el abundante crédito oficial. De acuerdo con la concepción ahora esbozada, era momento de que el sector público se retirase, delegando la financiación en los propios recursos de los empresarios y en el ahorro genuino. ${ }^{24}$

Asimismo, el capital internacional debía incrementar su participación en el financiamiento de los proyectos industriales que antes detentaba el sistema oficial. Sobre esa base lo manifestó Revestido en un artículo publicado por la revista Hechos e Ideas:

Muchas veces se ha hablado del carácter intervencionista del actual gobierno. Ello se funda, generalmente, en la serie de hechos que configuraron la primera etapa de sus realizaciones, cuando tuvo la imprescindible necesidad de asumir directamente sus posiciones de dirección y contralor para lograr rápidamente sus objetivos de independencia económica, soberanía política y justicia social. Pero cumplidos esos objetivos irrenunciables, la propia Constitución Justicialista de 1949, atribuye a la actividad privada una función fundamental en el desarrollo económico del país, con exclusión de la propiedad o explotación de aquellos sectores económicos que considera propios de la nación o del Estado en salvaguardia de la soberanía nacional, para luego agregar que la "iniciativa privada a través de las entidades representativas, no sólo está con el gobierno sino que, como ocurre con la organización de los trabajadores, está en el propio gobierno, es parte de él”. ${ }^{25}$

La idea de una fuerte presencia del Estado había quedado atrás y en este momento se interpelaba a diversos grupos económicos que en el pasado habían sido considerados hostiles o directamente enemigos de la patria.

También en esa línea el ministro defendió, a comienzos de 1955, la nueva postura gubernamental de intervención estatal en la economía. Sostenía que a partir de 1946, con la reforma financiera, el gobierno había

\footnotetext{
${ }^{23}$ Puntos, 1954, p. 11.

${ }^{24}$ Rougier y Fiszbein, Don, 2004.

${ }^{25}$ Revestido, "Reforma", 1954, p. 24.
} 
dejado el campo fértil para la consolidación de un mercado genuinamente inversor. Planteaba Revestido:

\begin{abstract}
Ahora, superado este primer paso con resultados halagüeños, por cuanto a la industrialización del país es hoy feliz realidad, es necesario propender a que las empresas busquen preferentemente en el ahorro privado los recursos para su desenvolvimiento a lo que agregó en clara oposición con la propia constitución de 1949 que las empresas que circunstancialmente debió conducir el Estado, pasaron a manos de la actividad privada, tal como lo expusiera en repetidas oportunidades el general Perón. ${ }^{26}$
\end{abstract}

El movimiento obrero debía esforzarse en todos los sectores de la productividad tratando de aumentarla de manera considerable; y de hecho una de las pocas acciones concretas que derivaron del anuncio del plan de acción fue el llamado en noviembre de 1954 a la realización del Congreso Nacional de la Productividad y el Bienestar Social, que tendría lugar en marzo del año siguiente, aunque tampoco lograría resultados importantes.

Los consumidores, por su parte (que en su mayoría eran trabajadores), ocuparon un lugar importante en la requisitoria presidencial, ya que debían defender sus ingresos controlando que los comerciantes respetasen los precios máximos fijados o los razonables cuando fueran de carácter "libre". Del mismo modo se tenían que negar a comprar y denunciar al Estado alguna situación anómala de precios para que este controlase y reprimiese "el agio y la especulación".

Para evitar caer en la compra de productos de precios elevados, se recomendó desarrollar e intensificar al máximo las actividades de las cooperativas, actividades propuestas en el segundo plan quinquenal. De esta forma el gobierno pretendía que la sociedad acompañara este proceso, que podríamos definir como una especie de "panóptico económico", donde el Estado controlaba mediante el poder de policía apoyado en la propia ciudadanía, que se encargaba de denunciar a los pequeños comerciantes. En esa línea, pero en la vida privada, se invitaba "imprescindiblemente" a que el consumidor realizara una conducta de "austeridad", eliminando los despilfarros y consumiendo lo que verdaderamente necesitaba.

Por el lado de los productores rurales, estos debían esforzarse por obtener una mejor utilidad de la tierra mediante una adecuada tecnificación de la explotación, proceso que a su vez permitiría obtener una producción de mejor calidad para la industria, el comercio y el consumo. Asimismo se debía propender a una mayor protección de los animales y vegetales para poder enfrentar las posibles contingencias climatológicas,

${ }^{26}$ Revestido, "Función”, 1955, p. 152. 
enfermedades o plagas. A la vez debían tender a la diversificación de la producción para minimizar hipotéticos riesgos económicos y asegurar el autoabastecimiento. ${ }^{27}$

El sector comercial también fue interpelado por Perón. Los comerciantes debían colaborar en la racionalización de la distribución de los productos para disminuir los costos. El presidente recomendaba que los comercios realizaran un sistema de autoservicio o self service para lograr contraer los precios de venta y mencionaba que era necesario crear conciencia en el comerciante minorista sobre las ventajas que representaba agruparse en "núcleos cooperativizados" para disminuir los gastos de comercialización. Por su parte, el presidente se comprometió a que el gobierno disminuiría los costos de los fletes distributivos de los artículos que partían de Buenos Aires hacia el interior. ${ }^{28}$

En lo que respecta a la labor gubernamental había un compromiso de colaboración con los demás actores sociales. El Estado garantizaría, por un lado, el abastecimiento interno de los productos que satisfacían el consumo y las necesidades vitales de la población y, por otro, el control de la fijación de precios a costos razonables y justificados. El organismo encargado de coordinar las políticas de abastecimiento y de precios era el Consejo Federal del Abastecimiento, con la colaboración de los gobiernos municipales, provinciales y las cooperativas de consumidores.

En relación con el comercio y abastecimiento exterior, siguiendo la línea del segundo proyecto, el plan de acción proponía mejorar la salida de productos que habían saturado el mercado interno mediante favorables fijaciones del tipo de cambio o incorporarlos en listas de convenios con terceros países, también planteaba intensificar la exportación de artículos manufacturados hacia mercados más beneficiosos. Este sistema no se daría mediante la política cambiaria, sino que debería ser proporcionado por un mejoramiento de orden interno con productos de mejor calidad, disminución de costos y una adecuada comercialización de los productos. ${ }^{29}$

Sobre esa base, el gobierno nacional prolongó la política crediticia moderada dando prioridad a las actividades que contribuyeran a aumentar la producción. Así, la ayuda al campo mediante préstamos procuró alcanzar la explotación integral y racional de los predios. Paralelamente, la asistencia financiera a la industria buscó el mejoramiento técnico mediante la racionalización en su organización, funcionamiento, equipamiento y reequipamiento. El fomento a las nuevas industrias de aprovechamiento de los recursos naturales fue central en el nuevo programa, así como también la ayuda al coope-

\footnotetext{
${ }^{27}$ Ibid., p. 9, y Perón, Plan, 1954.

${ }^{28}$ Revestido, "Función”, 1955, p. 11.

${ }^{29}$ Ibid., p. 17.
} 
rativismo y la intensificación del ahorro. A su vez, el Estado se comprometía a realizar las siguientes actividades: intensificar la explotación de tierras; proporcionar a los empresarios mineros el instrumento legal para proteger la actividad minera de exploración y explotación; profundizar los acuerdos nacionales e internacionales para la explotación petrolera; aumentar la disponibilidad de la energía eléctrica para la producción nacional; ahondar en las actividades de vialidad -vinculación caminera de los principales centros de producción con puertos, embarques ferroviarios y mercados de consumo-, de aguas para regadíos y de transporte; contención y reducción del gasto público disminuyendo la burocracia administrativa y manteniendo una política de austeridad y parsimonia en la aplicación del régimen impositivo; racionalizar el personal de cada organismo eliminando a los que no rindieran en la medida suficiente y no efectuar nuevos nombramientos a fin de reducir los gastos presupuestarios, y seleccionar a los nuevos funcionarios de acuerdo con su identificación con la doctrina nacional, el movimiento justicialista y su capacidad laboral. De este último ítem se desprende que los nombramientos burocráticos pasaron a ser -de forma excluyente- de acuerdo con el grado de pertenencia que se dispensaba al partido gobernante. Esto quedó implícito en el documento oficial demostrando que el sistema de lealtades era, en este periodo, la característica de ingreso al empleo público.

Para que el plan pudiera realizarse con el éxito esperado por el presidente y sus cuatro secretarios le otorgaron a la Secretaría de Prensa y Difusión la transmisión del programa para que la población en todo su conjunto tomase conocimiento sobre los motivos por los cuales el gobierno estaba vigilante de la economía nacional. Como cierre, la presidencia solicitó a los gobiernos de las provincias la comunicación de sus directores de prensa con la Secretaría de Prensa y Difusión para asesoramiento y coordinación de sus actividades. ${ }^{30}$

A pesar de que el plan estaba pensado en el corto plazo, temas como los relacionados con la colonización de tierras, planificación crediticia, el ahorro o la organización de las empresas como sociedades anónimas para cotizar en bolsa, señalaban la presencia de definiciones estratégicas más que coyunturales.

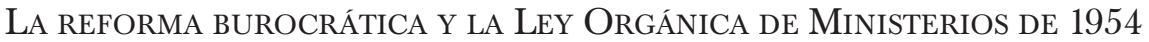

En 1951 finalizó el plan de gobierno o primer plan quinquenal, y los resultados no fueron los más satisfactorios para la conducción oficialista. En noviembre la ciudadanía volvió a las urnas con la novedad del voto feme-

${ }^{30}$ Ibid., pp. 21-29. 
nino, y le dio un nuevo mandato a la fórmula Perón-Quijano. Mientras se ponía en práctica el plan de emergencia de 1952 dirigido por Gómez Morales, desde el Ministerio de Asuntos Técnicos se diagramaba el segundo plan quinquenal, que comenzaría a regir en 1953.

Paralelamente a la ejecución del plan, en el mes de junio de 1952 se produjo la segunda reforma ministerial durante el peronismo, y los organismos económicos nuevamente sufrieron modificaciones sustanciales al igual que en 1949. Por la Ley 14.121/52 se creó una secretaría de Estado, el Ministerio de Asuntos Económicos, y se sustituyó el Ministerio de Economía por el de Comercio Exterior. En esta nueva transformación apareció Miguel Revestido al frente de Finanzas, y Alfredo Gómez Morales fue (re) designado en el nuevo ministerio.

A partir de esta modificatoria, el nuevo Ministerio de Asuntos Económicos fue el lugar donde se tomaron las medidas económicas más trascendentales y la figura de Alfredo Gómez Morales tomó una dimensión cada vez mayor en la decisión de políticas económicas. Sobre esta base burocrática, se delineó la política económica hasta 1954, momento en el que se constituyó una nueva reforma de los ministerios nacionales. ${ }^{31}$

El 19 de mayo de 1954 se realizó en la Casa de Gobierno una reunión de gabinete nacional. En esa oportunidad Perón les expuso a los ministros los planes gubernamentales tendentes a profundizar la transformación del Estado. A pesar de haber sorteado con éxito la crisis económica de 1952, el titular del poder ejecutivo consideraba que era necesaria una mayor intervención de la presidencia en lo relativo a la administración estatal. El motivo por el cual se realizó dicho encuentro fue informarles a los titulares de las diversas carteras las nuevas políticas que venía planificando desde tiempo atrás.

Las críticas que estaba recibiendo la gestión peronista para ese entonces por parte de la prensa y el arco político opositor se basaban en la falta de racionalidad o en lo pragmático de las resoluciones de los diferentes problemas económicos y sociales. Sin embargo, Perón les comentaba a sus ministros que el gobierno iniciado en 1946 había establecido un sistema burocrático completamente distinto en el país.

El presidente consideraba que se había alterado el sistema de compartimentación estatal con la creación de numerosos ministerios, y mencionó que a escala mundial, como producto de la planificación, varios países estaban en proceso permanente de inauguración de nuevas carteras. No obstante, Perón se encargó en reiteradas oportunidades de manifestarles a los ministros la necesidad de una reforma teórica-orgánica del gobierno y el Estado:

${ }^{31}$ Stawski, "Equipo”, 2012. 
Las organizaciones, para que sean objetivas, deben conseguir dedicarse a un objetivo y realizarlo. Ese es el concepto que yo tengo de la objetividad orgánica, primer y gran principio de la organización. El segundo principio ha de ser la simplicidad, vale decir, realizar esa función con los menores organismos posibles, ya que si son muy numerosos, se produce una obstrucción natural que trae siempre el concepto de esa excesiva burocracia donde los hombres andan estorbándose [...] El tercero de los principios es la estabilidad. Yo entiendo que ninguna organización puede rendir sus frutos acabadamente si no tiene un cierto grado de estabilidad [...] el otro principio de la organización que es la perfectibilidad. La perfectibilidad es antagónica con la estabilidad. Señores: indudablemente una organización perfecta estaría dentro de estos cuatro grandes principios. ${ }^{32}$

Así, para Perón la racionalidad burocrática era característica central de su gobierno. Para defender su gestión y no quedar en evidencia sobre los constantes recambios institucionales, el presidente mencionaba a la organicidad como precepto primario. Posteriormente presentó la reforma burocrática a través de la modificación de la cantidad de ministerios, para finalmente cerrar su discurso con el precepto de estabilización -tanto económica como burocrática- y perfección, todos ellos elementos en los cuales Perón se basaba para realizar la nueva modificación a la estructura burocrática.

Una vez presentado el problema a los ministros Perón explicó que el concepto perseguido tenía dos fases: una, la función de gobierno, de concepción y dirección, y, la segunda, de ejecución y verificación. Esto requería de dos diferentes grupos de organismos, el de concepción, constituido por un cuerpo de estudio bien centralizado, y el de ejecución, con un cuerpo de acción dividido en distintas partes para que pudieran ejecutar descentralizadamente las políticas recibidas. Finalmente el presidente les mencionaba a los ministros presentes: "Porque esto tampoco se puede realizar modificando de la mañana a la noche. Nosotros no podemos aparecer hoy con un ordenamiento que mañana reorganicemos completamente, porque haríamos un desaguisado terrible en el gobierno y en la administración [...] vamos a estudiarlo sin apuros y sin pensar en otra cosa que en la obligación que tenemos de construir el mejor instrumento de gobierno." ${ }^{33}$

No obstante la premura de Perón por informarles a los titulares de las carteras que se iba a realizar lenta y cuidadosamente el proyecto de reforma del Estado, a sólo un mes de la reunión de gabinete, el 25 de junio de 1954, se produjo la última reforma ministerial de los gobiernos peronistas, dejando en evidencia que lo presentado días antes no tenía significado. Es así que se observa que en tiempos de urgencia la contradicción entre el

${ }^{32}$ Perón, “Acuerdo”, 2002, pp. 243-252.

${ }^{33}$ Ibid. 
discurso y la acción por parte del propio Perón se hacía más recurrente y en menor plazo.

Dicha modificación fue aprobada por la Ley número 14.303 y el Decreto número 13.378/54, este último conocido como Reglamento de la Ley Orgánica de los Ministerios o Reglamento Orgánico de Ministerios (en adelante ROM) que tenía como propósito adecuar el funcionamiento de las carteras a las necesidades del país. El nuevo esquema era sumamente complejo en la implantación y en su ejecución, ya que las competencias de la gran mayoría de las agencias ministeriales desaparecieron, tomaron otras funciones o transfirieron algo de lo que disponían. A pesar de que desde la prensa oficialista el gobierno quería imponer la imagen de que la nueva modificación no constituía un cambio del sistema, sino una simple variación del régimen, es indudable que el nuevo escenario implicaba un nuevo ordenamiento técnico burocrático.

El proyecto se realizó sobre la base del artículo 84 de la Constitución Nacional de 1949 que facultaba al presidente de la nación a "determinar la denominación y los ramos de los ministerios, así como la coordinación de los respectivos despachos".

A través de este procedimiento se lograron establecer en orden de prioridades las relaciones directas de las tareas de ejecución en desarrollo con las funciones y con las estructuras que se encontraban bajo la dependencia del poder ejecutivo. Esta medida permitió, en primer lugar, aislar las dependencias orgánico-funcionales mínimas necesarias para el ejercicio de todas las funciones comprendidas en las competencias propias de la presidencia. Posteriormente se realizó un agrupamiento de estas agencias por actividades, resultado que derivó en el esquema ministerial con las funciones que debían desarrollar a futuro. ${ }^{34}$

Esta ley modificó la sancionada en 1949, y para el arco opositor significó una regresión de orden ideológico, ya que se volvía al estadio previo a ese año. La respuesta de los partidarios gubernamentales estaba dada por la idea de innovación y evolución constantes. Un artículo de la revista Hechos e Ideas de mediados de 1954 defendía de la siguiente manera la política gubernamental:

Si cambiamos, o si reformamos nuestra estructura ministerial, es precisamente, porque estamos afirmando una evolución constante y permanente del pueblo argentino. Casi me atrevería, y perdóneseme la audacia de una figura algo burda, a afirmar que las instituciones, los organismos estructurales y funcionales del gobierno se asemejan a los trajes que los padres compran a los hijos que están en la

34 "La nueva", 1954. 
pubertad: al año ya no sirven, porque los niños crecen rápidamente. La incomodidad del traje se debe al desarrollo del adolescente; la incomodidad que a veces se nota dentro del mecanismo gubernativo para el cumplimiento perfeccionado de una función de gobierno surge también de la evolución que se produce en el organismo estatal. ${ }^{35}$

En el aspecto teórico la nueva organización se encuadraba en los lineamientos del segundo plan quinquenal que proponía la racionalización de los organismos de gobierno. Este contexto servía como argumento defensivo para el gobierno, ya que consideraba la reforma como racional y planeada, pues llevaría a obtener por parte del Estado los más altos objetivos, a diferencia de las grandes reformas que sólo provocaban disturbios en el funcionamiento burocrático. De ahí que para el oficialismo haya sido un acierto la anterior reforma de descentralización de 1949, pues en su momento tuvo las ventajas de acercar a la función de gobierno la capacidad técnica para lograr sus objetivos.

Sin embargo, una vez superada esa instancia se creaban inconvenientes debido a la proliferación de organismos descentralizados que procedían de forma más o menos independiente, con pérdida del objetivo principal y entorpeciendo la capacidad de acción del gobierno. Al momento de la descentralización y de no poder controlar la entramada burocracia generada, era menester afianzar los organismos de coordinación. ${ }^{36}$

Este original reordenamiento dio una fisonomía final a la organización gubernamental distinguiendo, por un lado, los 16 ministerios y, por el otro, las cinco secretarías de presidencia. A los primeros les correspondía la tarea de ejecutar, y a las secretarías la de conducir. Este sistema permitía una capacidad operativa desconocida hasta el momento, ya que en cierto modo jerarquizaba los organismos ejecutivos estatales.

No obstante, esta disposición engendró tensiones entre las secretarías y los ministerios, dado que las primeras también podían ejecutar políticas relativas a su campo de acción. Se evidenciaron diversos conflictos entre estos grupos debido a la superposición de funciones. Sin lugar a dudas, esta modificación acrecentaba el poder de las secretarías por sobre los ministerios. ${ }^{37}$

De gran importancia en la Ley fue el régimen que imponía para "la racional coordinación de los organismos". El artículo 6 refería: "los despachos de los negocios de la nación serán coordinados atendiendo a la doctrina nacional y a los planes de gobierno que en ella se fundamentan

\footnotetext{
${ }^{35}$ Juárez, "Nueva”, 1954, p. 31.

${ }^{36} \mathrm{Ibid}$

${ }^{37}$ Oyuela, "Historia", 1976, p. 53.
} 
y mediante los acuerdos, órganos y consejos de coordinación general o específica que determine el poder ejecutivo". Es en este artículo donde se pueden distinguir las modificaciones más importantes en relación con la nueva estructura, ya que aparecen "acuerdos, órganos y consejos" que en definitiva recogía de organismos preexistentes, pero dejando de manifiesto que la dirección iba a ser llevada a cabo por Perón. Así, y para prevenir una especie de "autonomía decisoria" por parte de los organismos descentralizados, fue coherente con la línea gubernamental, en vista de reducción de riesgos, crear o afianzar las agencias de coordinación.

Tres fueron los aspectos que persiguió en teoría la Ley. El primero vinculado a la concepción de la acción del gobierno, el segundo a la ejecución del segundo plan quinquenal y el tercero a controlar su implementación. Sobre esta base los nuevos ministerios secretarías de Estado quedaron estructurados de la siguiente manera: Relaciones Exteriores y Culto; Interior y Justicia; Trabajo y Previsión; Educación; Asistencia Social y Salud Pública; Comunicaciones; Obras Públicas; Transportes; Hacienda; Agricultura y Ganadería; Industria; Comercio; Finanzas; Ejército; Marina, y Aeronáutica. Por el lado de las secretarías de presidencia figuraban Asuntos Económicos, Asuntos Políticos, Asuntos Exteriores, Defensa Nacional y Asuntos Técnicos. ${ }^{38}$ De esta nueva reestructuración se desprende una predominancia de organismos correspondientes al área económica sobre lo político-social, aspecto totalmente inédito hasta ese momento.

Los ministerios de Asuntos Económicos, Defensa Nacional, Asuntos Técnicos y Asuntos Políticos fueron suprimidos, dada la naturaleza de las actividades que realizaban, ya que en su breve experiencia habían demostrado, siempre según el gobierno, la imposibilidad de conjugar en el gabinete las tareas de concepción y de planificación de objetivos con aquellas de ejecución de dichos planes. Perón consideró que no podían determinarse como ministerios aquellas agencias que tenían como objetivo central la función de concebir, planificar, coordinar y racionalizar.

También se establecieron, por Decreto número 12.171 del 22 de julio de 1954 , las funciones de los cuatro ex ministerios que no ingresaron en el ROM debido a que carecían de la tarea de ejecución. En relación con la Secretaría de Asuntos Económicos se le atribuyó la tarea de presidir el Consejo Económico Nacional y "asistir al presidente en todo lo inherente al afianzamiento de la independencia económica y al desarrollo de la economía social y en particular las actividades económicas y financieras de la nación". ${ }^{39}$

Las otras secretarías cumplían funciones similares a las descritas pero obviamente de acuerdo con su campo de acción. Como se puede obser-

\footnotetext{
${ }^{38}$ Juárez, "Nueva", 1954, pp. 40 y ss.

39 "Reglamento", 1954.
} 
var, la Secretaría de Asuntos Económicos ejerció en poco más de un año de vida funciones de carácter técnico, basada su existencia en los estudios, la racionalización o la planificación. De esta forma había quedado diagramada la nueva estructura ejecutiva de Argentina. ${ }^{40}$ Por un lado y bajo un férreo control de la presidencia, los ministerios, encargados de ejecutar las políticas dictadas por Perón y, por otro, las secretarías, órganos facultados dependientes del poder ejecutivo con la tarea de realizar la coordinación y conducción de la política oficial.

Los nuevos secretarios del poder ejecutivo debían, según dicho decreto: "asistir al presidente de la nación en el ejercicio de sus funciones vinculadas a la conducción del gobierno y particularmente en todo lo relativo a la coordinación del despacho de los negocios de la nación". Asimismo, estas dependencias debían reunir y apreciar la información necesaria para el poder ejecutivo; proponer planes de gobierno; coordinar la acción de los ministerios y efectuar el cumplimiento de las resoluciones y planes en vigencia, informar al presidente y proponer las medidas a adoptar. ${ }^{41}$

Concomitantemente los secretarios integraban el novedoso Consejo de Gobierno, que era convocado en acuerdo de gobierno por la presidencia cada vez que lo hubiera considerado necesario. Por su parte, el Consejo de Gabinete estaba integrado por los secretarios, ministros y el presidente, y realizaba la coordinación del despacho de los negocios de la nación, armonizando las tareas de ejecución descentralizada. ${ }^{4}$

En relación con los titulares de los ministerios, Miguel Revestido y Pedro Bonanni, continuaron al frente de Finanzas y Hacienda, respectivamente, y Gómez Morales fue designado secretario de Asuntos Económicos. Formalmente quedó reflejada la supremacía de que disponía esta secretaría por encima de las carteras de Finanzas y Hacienda.

En el pasado no estaba expresada legalmente esta situación -ya que técnicamente todos los ministerios disponían de la misma jerarquía-, pero con la reforma burocrática quedó cristalizada esta novedosa ordenación que colocaba en la cima de la decisión económica a la Secretaría de Asuntos Económicos. La nueva oficina mantuvo personal, sede y los bienes del ex ministerio homónimo. Las atribuciones de esta secretaría eran las mismas que cuando tenía rango de ministerio, pero incrementaba sus funciones aún más, ya que disponía de la presidencia del Consejo Económico Nacional y realizaba todas las proposiciones de la política económica a la presidencia. ${ }^{43}$

\footnotetext{
40 "La nueva", 1954, p. 273.

${ }^{4}$ Ibid.

42 "Las secretarías", 1954, p. 113.

43 Juárez, "Nueva", 1954, p. 42.
} 
Por su parte, el Ministerio de Finanzas terminó cediendo gran parte de su actuación, y con el ROM sus funciones eran:

asistir al presidente de la nación en la ejecución de la política bancaria, monetaria, cambiaria, mercado de valores mobiliarios, seguros y reaseguros y en particular lo inherente a: a) la defensa del valor de la moneda, $b$ ) regulación del crédito y los medios de pago, $c$ ) autorización y fijación de tasa de interés bancario, $d$ ) régimen de fiscalización de la actividad bancaria del mercado de valores mobiliarios, de los seguros y reaseguros, $e$ ) fijación de los tipos de cambio, $f$ ) elaboración y ejecución del presupuesto de divisas, $g$ ) intervención de acuerdos, convenios y arreglos internacionales de carácter financiero y comercial, $h$ ) operaciones de crédito interno y externo, de empréstitos públicos, i) acuñación de moneda e impresión de billete, $j$ ) formulación y ejecución de los planes de ahorro, $k$ ) colonización en tierras de origen privado. ${ }^{44}$

Uno de los nuevos ministerios que se creó fue el de Comercio en reemplazo del de Comercio Exterior. Continuó al frente del mismo Antonio Cafiero, pero bajo la órbita de la Secretaría de Asuntos Exteriores. En esta nueva burocratización, el IAPI sufrió restructuraciones y continuó, con marchas y contramarchas, realizando las funciones detentadas desde 1949, año que pasó a depender de Economía. Ya en 1949 había sufrido la pérdida de su autonomía y de su capacidad de gestión con la reforma ministerial, pero sería en 1954 cuando abandonaría las funciones de organismo comercializador de la producción agropecuaria que venía desarrollando desde 1946.

Con motivo de la ROM se le incorporó la nueva cartera el Instituto Nacional de Carnes -Ley número 14.379 de octubre de 1954-, así como también la Dirección Nacional de Granos y Elevadores -Ley número 14.378- y las gerencias de productos agrícolas y de productos ganaderos que provocaban superposición de funciones y dividían innecesariamente los aspectos comerciales y técnicos del ministerio. Es así que tanto el Instituto de Carnes como la dirección pasaron a ser entidades descentralizadas del Ministerio de Comercio y comenzaron a ejercer por cuenta y orden del IAPI las funciones que antes desempeñaba este.

Con esta medida se intentó una especialización de funciones con el objetivo de asegurar la mayor eficiencia para el cumplimiento de los fines propuestos por el Estado en la producción agropecuaria, descentralizando el IAPI para alcanzar dichos fines. En cuanto a la importación, el IAPI prosiguió la tendencia de abandonar su accionar en los rubros que en ese momento podían estar en manos de importadores privados. ${ }^{45}$ De esta forma

\footnotetext{
${ }^{44}$ Ibid.

${ }^{45}$ Memoria, 1955, pp. 12-13.
} 
el propio gobierno le daba a una de las instituciones más emblemáticas del primer peronismo el golpe final, ya que desde 1949 había comenzado una pérdida paulatina de su accionar para terminar de cumplir sus funciones primarias e iniciales a fines de 1954 .

Esta nueva reestructuración se basaba en la idea de Perón de que las tres fases que caracterizaban a todo gobierno eran la conducción, la ejecución y el contralor. Estos principios se trataron en el citado artículo 6 de la Ley, referente a la conexión de los despachos ministeriales, con el objetivo de afirmar que la coordinación tenía que ser producto de órganos que intervinieran no en la ejecución, sino en la concepción de la acción de los planes. Estaba subyacente en el pensamiento gubernamental la idea de que la organización debía estar por esencia empíricamente fundamentada en los datos y razones suministrados por el accionar, por la vivencia real de la jornada administrativa, es decir, por la experiencia. ${ }^{46}$

La reforma de las agencias económicas dejó al descubierto una clara necesidad del poder ejecutivo en cuanto a profundizar la reforma burocrática para darle mayor poder de decisión a la presidencia. Se observa en este periplo que a comienzos de 1946 Perón solicitaba reiteradamente la creación de nuevos ministerios, hecho que formalmente se produjo en 1949. Una vez producida la reforma y consolidado el equipo económico con la implementación de una gran cantidad de carteras, entre ellas el Ministerio de Finanzas y el de Economía, el presidente realizó una nueva transformación de las agencias económicas con la creación del Ministerio de Asuntos Económicos en 1952.

Allí se colocó a Alfredo Gómez Morales, en coordinación con los demás ministros del equipo económico, para dotar de una mayor racionalidad y eficiencia a la política económica. Sin embargo, nuevamente en 1954 se volvería a una situación similar a la inicial con el objetivo de incrementar el poder de decisión de la presidencia con la jerarquización de las secretarías por encima de los diversos ministerios.

El flamante esquema que concentró en pocos organismos y personajes las decisiones de índole financiera fue el resultado del camino que atravesó el Estado en la sanción de políticas económicas. El contexto de reforma colocó la figura presidencial -junto con cinco secretarios- por encima de los elencos ministeriales que se encargaban asimismo de consolidar la doctrina peronista. Y sería, una vez más, sobre la base de esta nueva estructura burocrática que un nuevo plan de ajuste se impondría durante el gobierno de Juan Perón. 


\section{REFLEXIONES FINALES}

A partir de 1949, y con más fuerza desde 1952, el gobierno peronista avanzó en una serie de modificaciones importantes en las definiciones económicas, en gran medida motivadas por las dificultades que se presentaron en el frente externo. No obstante, esas alteraciones se realizaron tratando de mantener su principal base social de apoyo -los trabajadores-, a la vez que ganar nuevos sectores del empresariado rural y urbano. Ello supuso impulsar un "nuevo trato" entre capital y trabajo y una forma y participación diferentes del Estado en su articulación con los actores sociales. Si en los primeros años de gobierno la intervención pública era de importancia clave para los objetivos propuestos, luego de la crisis el Estado se reservaba una participación menor, aunque de todos modos importante, como orientador de la política económica y coordinador de las demandas sectoriales.

El Plan de Acción de Equilibrio para la Economía Nacional de octubre de 1954 reflejó con claridad los nuevos lineamientos y objetivos que tendían a "racionalizar" la influencia estatal y la dinámica económica. Con el convencimiento de que la injerencia pública generaba fuertes distorsiones, los funcionarios impulsaban ahora una participación menor del Estado -y en términos actuales una mayor calidad de la intervención estatal- en los mecanismos determinantes de los salarios, en las inversiones y en la orientación del crédito, en el control de las importaciones y exportaciones, entre otras; en suma, menos Estado y más mercado pasaba a ser la consigna del momento, en línea con la formación ortodoxa de los principales conductores en materia económica.

Pero estos cambios suponían necesariamente modificaciones en la esfera burocrática, particularmente importantes en aquellas dependencias y organismos vinculados a la gestión económica. En otras palabras, para su éxito la racionalización económica pretendida debía sustentarse en una racionalización burocrática a la vez que en una mayor centralización del poder de decisión. Estas políticas fueron impuestas gracias a la incorporación de elencos técnicos al Estado y a las modificaciones de la estructura burocrática, que tuvo mayor impulso con la nueva Constitución de 1949.

Una vez encarada la puesta en marcha del segundo plan quinquenal y en vista de afianzar el control del ejecutivo sobre los ministerios se decidió, en 1954, continuar con la reforma del Estado en una línea clara de ensayo y error. Las múltiples agencias económicas creadas a partir de 1949 quedaron subordinadas a los ministerios de gestión económica, y estos a su vez bajo la supervisión de la Secretaría de Asuntos Económicos, dirigida por Gómez Morales.

La Ley Orgánica de Ministerios de 1954, encuadrada dentro de los objetivos de racionalización del gobierno, promovió que las carteras dispu- 
sieran solamente de la facultad de ejecutar las directivas de la presidencia. Este objetivo contrastaba rotundamente con el de la reforma de 1949, que había impulsado la creación de una mayor cantidad de ministerios para lograr los múltiples objetivos gubernamentales.

En todo caso, y como medio para asegurar cierto grado de control, Perón decidió subordinar las agencias económicas a la nueva secretaría. Así, y una vez que se estableció este sistema, Gómez Morales dispuso de un real poder decisorio a escala nacional. Las dependencias anteriormente trascendentes, como el Consejo Económico Nacional, el Banco Central, el IAPI y las diversas comisiones, perdieron autonomía al quedar supeditadas a las directivas de la Secretaría de Asuntos Económicos. Ese proceso de ordenamiento y concentración de la toma de decisiones era, en definitiva, sustancial para consolidar los nuevos objetivos de racionalización económica que el gobierno se propuso en sus últimos años.

\section{BiBLIOGRAFÍA}

Belini, Claudio, La industria peronista, Buenos Aires, EDHASA, 2009.

Berrotarán, Patricia M., Del plan a la planificación. El Estado durante la época peronista, Buenos Aires, Imago Mundi, 2003.

"Estado, planificación y peronismo. Los debates 1940 a 1946", Anais Eletrônicos do VIII Encontro Internacional da Associação Nacional de Pesquisadores e Professores de História das Américas, Vitória, 2008, en <http://anphlac.org/upload/anais/ encontro8/patricia_berrotaran.pdf $>$. [Consulta: 6 de julio de 2012.]

Bitrán, Rafael, El Congreso de la productividad, Buenos Aires, El Bloque Editorial, 1994.

Brennan, James y Marcelo Rougier, The Politics of National Capitalism Peronism and the Argentine Bourgeoisie, 1946-1976, Pensilvania, Pennsylvania State University Press, 2009.

CAmpione, Daniel, Prolegómenos del peronismo. Los cambios en el Estado nacional, 1943-1946, Buenos Aires, Fundación de Investigaciones Sociales y Políticas, 2003.

Castellani, Ana y Flavia Llanpart, "Debates en torno a la calidad de la intervención estatal", Papeles de Trabajo, Universidad Nacional de General San Martín, año 6, núm. 9, primer semestre, junio de 2012, pp. 155-177.

Fernández, Fabián, La huelga metalúrgica de 1954, Buenos Aires, Centro Cultural de la Cooperación, 2005.

Ferrer, Aldo, Crisis y alternativas de la política económica argentina, Buenos Aires, Fondo de Cultura Económica, 1977.

Gerchunoff, Pablo y Lucas Llach, El ciclo de la ilusión y el desencanto, Buenos Aires, Ariel, 1998. 
Jáuregui, Aníbal, "Prometeo encadenado: los industriales y el régimen peronista" en Patricia Berrotarán, Aníbal Jáuregui y Marcelo Rougier, Sueños de bienestar en la nueva Argentina, Buenos Aires, Imago Mundi, 2004, pp. 47-106.

JuÁREZ, CARLOS, "La nueva restructuración de los ministerios", Hechos e Ideas, año XV, núms. 122-123, junio-julio de 1954, Buenos Aires.

"La nueva Ley Orgánica de los Ministerios del Poder Ejecutivo de la Nación”, Horizontes Económicos, año X, núm. 105, junio, 1954, Buenos Aires.

"Las secretarías del poder ejecutivo", Economía y Finanzas, 29 de julio de 1954, Buenos Aires.

Memoria anual del Instituto Argentino para la Promoción del Intercambio. Ejercicio 1954, Ministerio de Comercio de la Nación, Buenos Aires, 1955.

Novick, Susana, IAPI: auge y decadencia, Buenos Aires, Centro Editor de América Latina, 1986.

Oyuela, Juan, "Historia de la planificación argentina", Cuadernos de Planeamiento, año I, núm. I, diciembre de 1976, Buenos Aires, pp. 50-51.

OSZlaK, Oscar, Políticas públicas y regímenes politicos: reflexiones a partir de algunas experiencias latinoamericanas, Buenos Aires, Centro de Estudios de Estado y Sociedad, 1980. (comp.), Teorías de la burocracia estatal: enfoques críticos, Buenos Aires, Paidós, 1984.

Perón, Juan Domingo, Plan de acción para el equilibrio de la economía nacional, Buenos Aires, Presidencia de la Nación-Secretaría de Prensa y Difusión, 1954.

"En el acuerdo de ministros. 19 de mayo de 1954", Obras completas, Buenos Aires, Docencia, 2002.

, "En la primera Convención convocada por la Confederación General Económica. 2 de julio de 1954", Obras completas, Buenos Aires, Docencia, 2002. , "En el Salón Blanco de la Casa de Gobierno sobre la marcha de la economía. 1 de octubre de 1954", Obras completas, Buenos Aires, Docencia, 2002.

Puntos de vista del gobierno y el Estado nacional para iniciar, de inmediato, la ejecución del "Plan de acción para el equilibrio de la economía nacional", Buenos Aires, Presidencia de la Nación, Secretaría de Prensa y Difusión, 1954.

Prol, Mercedes, "Peronismo, elites políticas e instituciones de gobierno, 1943-1952" en IX Jornadas Interescuelas, Universidad Nacional de Córdoba, 24 a 26 de septiembre de 2003, CD-ROM.

RAPOPORT, MARIO, Historia económica, política y social de la Argentina (1880-2000), Buenos Aires, Sudamericana, 2000. y Claudio Spiguel, Estados Unidos y el peronismo. La política norteamericana en la Argentina, 1945-1955, Buenos Aires, Grupo Editor Latinoamericano, 1994.

"Reglamento de la Ley núm. 14.303 Orgánica de los Ministerios (ROM)”, Horizontes Económicos, año X, núm. 107, agosto, 1954, Buenos Aires.

ReIn, RAANAN, Juan Atilio Bramuglia. Bajo la sombra del líder, la segunda línea del liderazgo peronista, Buenos Aires, Lumiere, 2006. 
Revestido, Miguel, "La reforma económico social del gobierno y la Bolsa de Comercio", Hechos e Ideas, año XV, núms. 122-123, junio-julio de 1954, Buenos Aires. "La función del Mercado de Valores Mobiliarios. El ahorro privado en el fomento de la industria", Hechos e Ideas, año XV, núm. 130, febrero de 1955, Buenos Aires.

Rougier, MARCELO, El mercado de valores durante el peronismo. Las dificultades para conformar un ámbito de capitalización empresarial (1944-1955), mimeo., 2011. , La economía peronista, Buenos Aires, Sudamericana, 2012.

y Martín Fiszbein, "De don Derrochín a maese Ahorrín. El fomento del ahorro durante la economía peronista” en PATricia BERrotarán, ANíbAl JÁureGUi y Marcelo Rougier (comps.), Sueños de bienestar en la nueva Argentina. Estado y políticas públicas durante el peronismo 1946-1955, Buenos Aires, Imago Mundi, 2004.

Schiavi, Marcos, "Algunas consideraciones sobre poder obrero y productividad en el primer peronismo: la mirada de los industriales metalúrgicos (1936-1955)" en Daniel Dicósimo y Silvia Simonassi (comps.), Trabajadores y empresarios en la Argentina del siglo XX, Rosario, Prohistoria, 2011.

SCHNEIDER, BEN R., "Las relaciones entre el Estado y las empresas y sus consecuencias para el desarrollo: una revisión de la literatura reciente", Desarrollo Económico, Instituto de Desarrollo Económico, vol. 39, núm. 153, abril-junio de 1999, pp. 45-75.

STAWSKI, MARTín, "Del equipo de asalto a la consolidación: Estado, economía y elites durante el primer peronismo, 1946-1955" en MARIANo PlotKin y EdUARdo Zimmermann (comps.), Las prácticas del Estado. Política, sociedad y elites estatales en la Argentina del siglo XX, Buenos Aires, EDHASA, 2012.

"De los Consejos al Ministerio. La gestión de la economía y las transformaciones de la trama burocrática estatal bajo el peronismo (1946-1955)", tesis de doctorado, Argentina, Universidad de Buenos Aires, 2012. 